\title{
Alpha-fetoprotein and carbohydrate antigen 19-9 producing advanced adenocarcinoma of renal pelvis and ureter
}

\author{
Kai Yang, MD; Xiang-Yi Zheng, MD; ${ }^{*}$ Yan-Li Wang, MD; ${ }^{+}$Kui Zhao, MD; ${ }^{s}$ Hai Jiang, MD;
}

*Department of Urology, the First Affiliated Hospital, School of Medicine, Zhejiang University, Zhejiang Province, China; 'Department of Pathology, the First Affiliated Hospital, School of Medicine, Zheijang University, Zhejiang Province, China; §PET Center, the First Affiliated Hospital, School of Medicine, Zhejiang University, Zhejiang Province, China

Cite as: Can Urol Assoc J 2013;7(11-12):e750-3. http://dx.doi.org/10.5489/cuaj.1544 Published online November 8, 2013.

\section{Abstract}

Tumour markers producing primary adenocarcinoma of upper urinary tract is extremely rare. We report a case of advanced adenocarcinoma of renal pelvis and ureter with highly elevated serum levels of alpha-fetoprotein (AFP) and carbohydrate antigen 19-9 (CA19-9). This 66-year-old man was diagnosed with left renal pelvic and ureteral tumours with para-aortic lymph node swelling, with no evidence of abnormality in his digestive or reproductive system. He was successfully treated with left nephroureterectomy and lymph node dissection followed by gemcitabine/carboplatin chemotherapy and the serum levels of AFP and CA19-9 decreased to normal. Pathological examination revealed a moderately or poorly differentiated intestinal-type adenocarcinoma with paraaortic lymph node metastasis. The patient was followed up for 11 months after surgery without recurrence.

\section{Introduction}

Alpha-fetoprotein (AFP) is a fetal serum protein which is considered as a tumour marker of several types of cancer, such as hepatocellular carcinoma (HCC), yolk-sac tumour and other gonadal neoplasms. Carbohydrate antigen 19-9 (CA19-9) is another well-known tumour marker which is elevated in many adenocarcinomas of digestive system, especially pancreatic tumours. Tumours arising from the epithelium of upper urinary tract seldom produce AFP or CA19-9. Moreover, only about $1 \%$ of malignancies of renal pelvis or ureter correspond to adenocarcinoma. ${ }^{1}$ Therefore, AFP or CA19-9 producing adenocarcinoma of renal pelvis or ureter is extremely rare. To our knowledge, only several cases with either AFP or CA19-9 positive have been reported..$^{2-14}$

We report a case of advanced adenocarcinoma of renal pelvis and ureter which produced AFP and CA19-9 simul- taneously. This seems to be the first documentation of both AFP and CA19-9 producing adenocarcinoma of upper urinary tract.

\section{Case report}

A 66-year-old Chinese man was admitted to hospital complaining of facial edema for 1 week. Urinalysis revealed 30 erythrocytes per high-power field, but no leukocytes or casts. Blood examinations showed extremely elevated serum levels of AFP $(407.8 \mathrm{ng} / \mathrm{mL}$, normal $<20.0 \mathrm{ng} / \mathrm{mL})$ and CA19-9 ( 2739.6 units $/ \mathrm{mL}$, normal $<37.0$ units $/ \mathrm{mL}$ ). Abdominal ultrasonography revealed a $7.6 \times 1.4-\mathrm{cm}$ mass in the lower part of the left ureter and a $3.3 \times 3.4-\mathrm{cm}$ mass in the left renal pelvis with severe hydronephrosis. Subsequent contrastenhanced computed tomography (CT) also demonstrated these tumours of both left renal pelvis (Fig. 1, parts A and B) and ureter (Fig. 1, part C) with a swelling para-aortic lymph node (Fig. 1, part D). However, 3 cytologic examinations of urine did not find any atypical cells. To figure out whether there was another tumour from the digestive or reproductive system, which might be responsible for the elevated serum levels of AFP and CA19-9, the patient underwent a positron emission tomography-CT (PET-CT) examination. After that, PET-CT confirmed the results of contrast-enhanced CT and suggested that these tumours were probably malignant (Fig. 2). Moreover, there was no evident abnormality in the liver, pancreas, gallbladder, or reproductive system.

Then, a left nephroureterectomy and lymph node dissection were performed. The subsequent pathological diagnosis was moderately or poorly differentiated intestinal-type adenocarcinoma of left renal pelvis and ureter (Fig. 3, part A) with para-aortic lymph node metastasis. Immunohistological analysis of the tumour demonstrated that cancer cells were positive for AFP (Fig. 3, part B) and CDX2 (Fig. 3, part C) and negative for CK7, CGA and SYN. After that, chemotherapy with gemcitabine and carboplatin was 

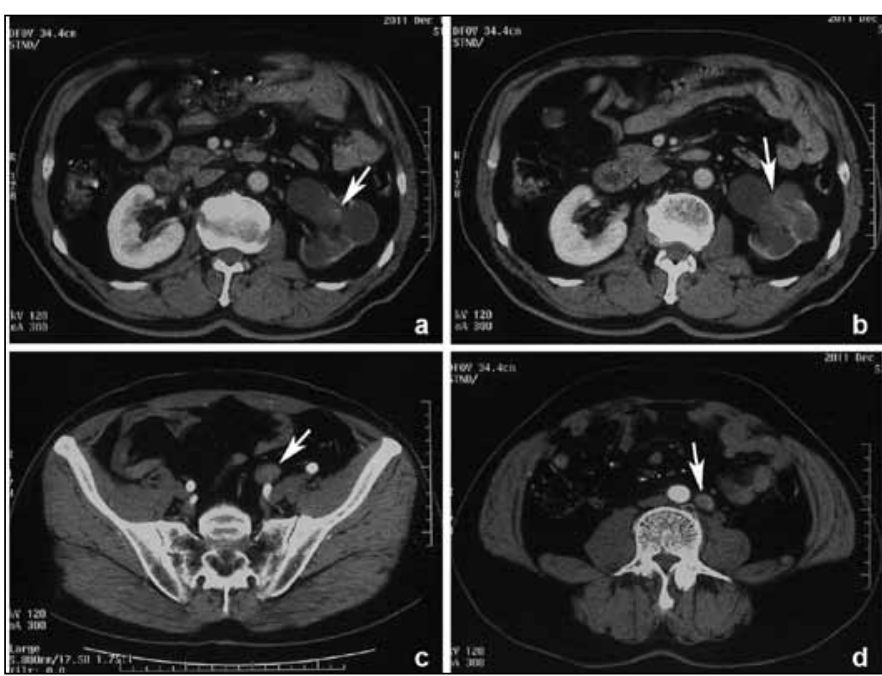

Fig. 1. Contrast-enhanced computed tomography demonstrates (a, b) left pelvic tumour; (c) ureteral tumour; and (d) a swelling para-aortic lymph node (arrow). initiated. The serum levels of AFP and CA19-9 decreased immediately after surgery and remained normal during the follow-up (Fig. 4). The patient was carefully followed and no sign of recurrence was observed for 11 months. The patient is currently fine.

\section{Discussion}

AFP or CA19-9 producing adenocarcinoma of upper urinary tract is rare. To our knowledge, only 3 cases of AFP producing adenocarcinoma ${ }^{2-4}$ and 10 CA19-9 positive cases $^{5-14}$ have been reported. However, there is no report on both AFP and CA19-9 producing tumour of the renal pelvis or ureter. In the present case, the unique feature of this patient was the highly elevated serum levels of AFP and CA19-9, with no abnormality in the digestive or reproductive system. Although we only performed the immuno-histochemical staining for

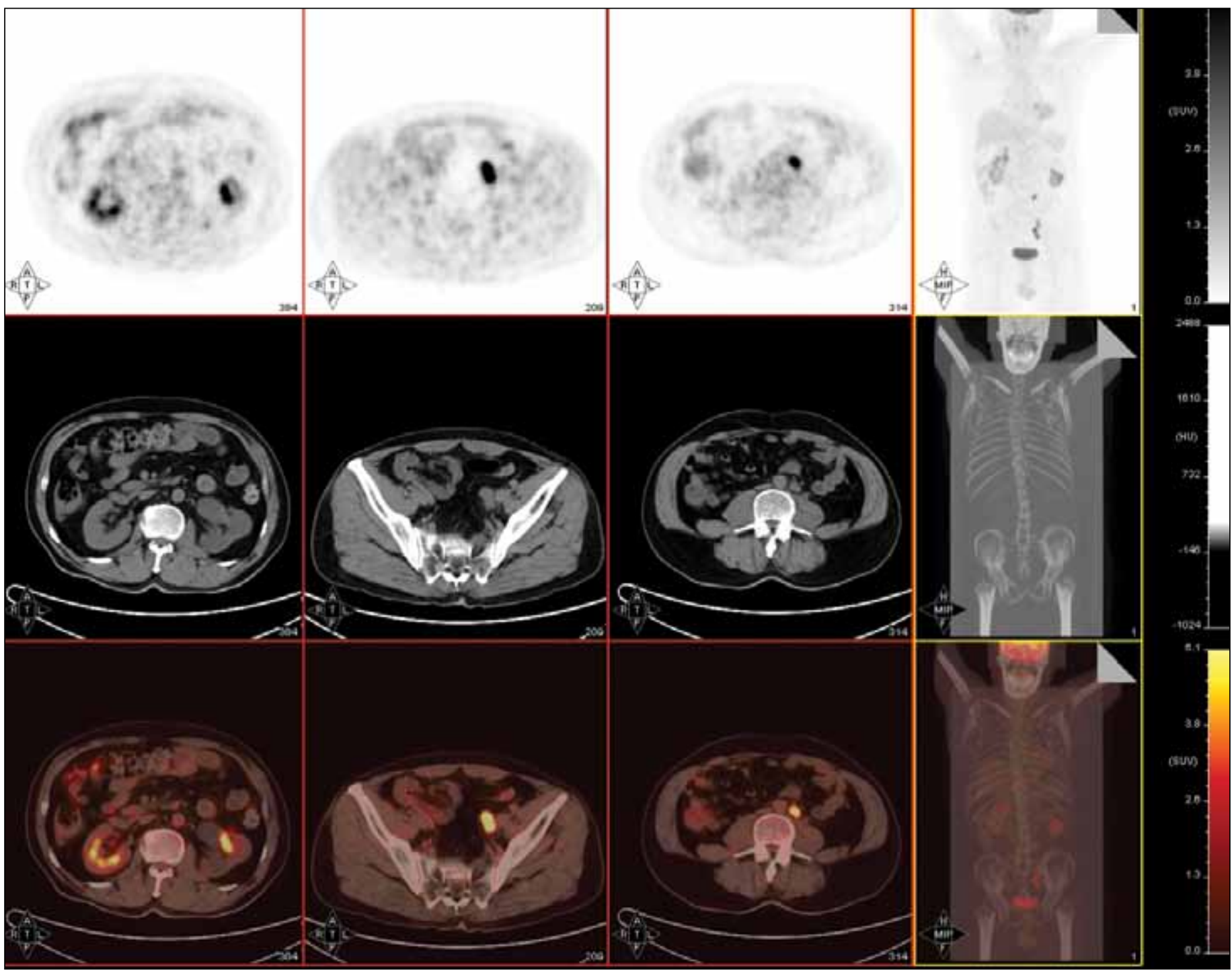

Fig. 2. Positron emission tomography-computed tomography confirms the tumours in the left renal pelvis and ureter and suggests cancer metastasis in the swelling para-aortic lymph node. 


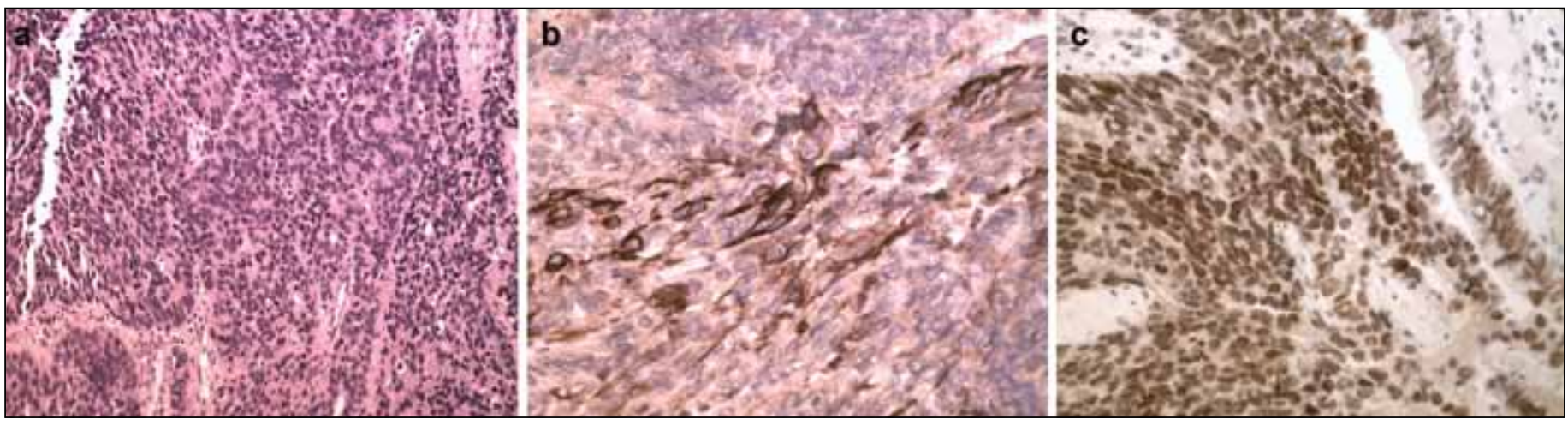

Fig. 3. (a) Pathological examinations revealed that the left renal pelvic and ureteral tumours are intestinal-type adenocarcinoma (hematoxylin-eosin $\times 200)$. (b) Immuno-histochemical staining for alpha-fetoprotein showed positive cytoplasmic staining in the tumour cells $(\times 400)$. (c) Immuno-histochemical staining for CDX2 showed positive cytoplasmic staining in the tumour cells $(\times 400)$.

AFP, which was actually positive, the normalization of both 2 tumour markers after surgery and chemotherapy also supported that contention that elevated AFP and CA19-9 originated from the primary renal pelvic and ureteral malignancy.

There is no established regimen of chemotherapy for metastatic primary adenocarcinoma of the urinary tract. Other studies show that paclitaxel plus carboplatin was more effective than the methotrexate, vinblastine, doxorubicin and cisplatin (MVAC) chemotherapy, ${ }^{9}$ and it was applied in the treatment of some cases subsequently. ${ }^{11,13}$ In our case, we tried gemcitabine plus carboplatin for adjuvant chemotherapy after surgery, and no sign of recurrence and severe side-effects were observed for 11 months, suggesting that this regimen of chemotherapy might be used to

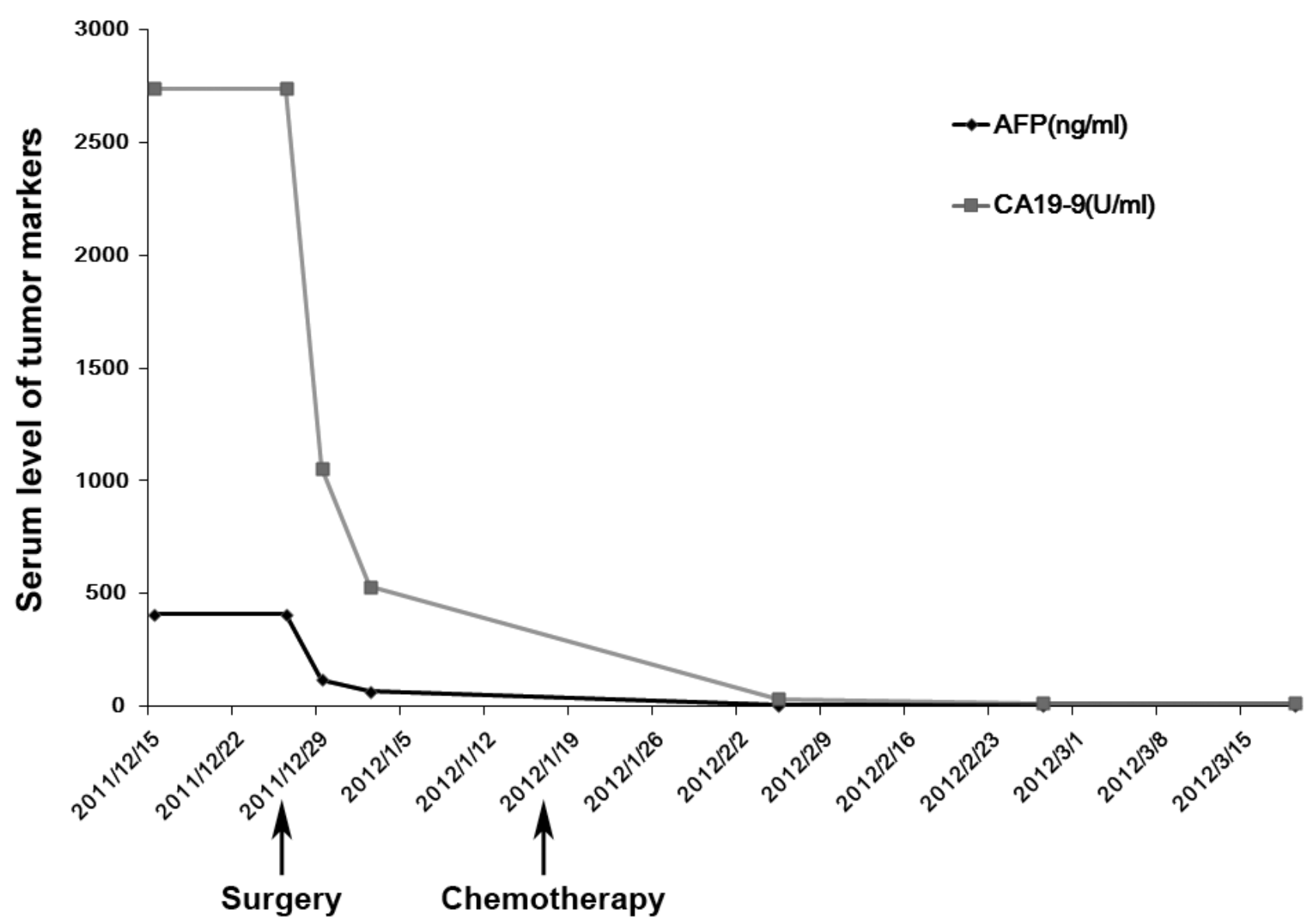

Fig. 4. The follow-up of the serum levels of alpha-fetoprotein and CA19-9. AFP: alpha-fetoprotein. 
treat patients with metastatic primary adenocarcinoma of the upper urinary tract.

The prognosis of these tumour markers producing adenocarcinoma seems relatively good. Although several cases did not receive adjuvant chemotherapy or radiotherapy and died of metastasis, most patients with AFP or CA19-9 producing tumours were alive without recurrence for at least 6 months. $^{2-14}$

\section{Conclusion}

Adenocarcinoma of upper urinary tract, which produces AFP and CA19-9 simultaneously, is rare. Further investigation is needed to delineate the nature of this type of tumour and the proper course of treatment.

Acknowledgments: This study was supported by grants from the National Natural Science Foundation of China (Grant No. 81 101718) and the National Key Clinical Specialty Construction Project of China.

Competing interests: Dr. Yang, Dr. Zheng, Dr. Wang, Dr. Zhao and Dr. Jiang all declare no competing financial or personal interests.

This paper has been peer-reviewed.

\section{References}

1. Grabstald H, Whitemore WF, Melamed MR. Renal pelvic tumors. JAMA 1971;218:845-54. http:// dx.doi.org/10.1001/jama.1971.03190190031006
2. Ishikura H, Ishiguro $\mathrm{T}$, Enatsu $\mathrm{C}$, et al. Hepatoid adenocarcinoma of the renal pelvis producing alpha-fetoprotein of hepatic type and bile pigment. Cancer 1991;67:3051-6. http://dx.doi.org/10.1002/10970142(19910615)67:12<3051::AID-CNCR2820671220>3.0.C0;2-C

3. Hosomi M, Sagawa S, Kotou Y. Alpha-fetoprotein-producing adenocarcinoma of the ureter. Urol Int 1992;48:226-7. http://dx.doi.org/10.1159/000282339

4. Sakata Y, Onishi T, Yamada Y, et al. -Fetoprotein producing renal pelvic and ureter tumor. J Urol 2001;166:1830. http://dx.doi.org/10.1016/50022-5347(05)65689-3

5. Haitel $A$, Wiener HG, Susami M. Primary adenocarcinoma of the ureter. Case report with immunohistochemical characterization. Pathol Res Pract 1996;192:81-5. http://dx.doi.org/10.1016/S03440338(96)80140-3

6. Imaki H, Wakabayashi Y, Kushima R, et al. Primary adenocarcinoma of the ureter producing carbohydrate antigen 19-9. J Urol 1996;156:1437. http://dx.doi.org/10.1016/S0022-5347(01)65612-X

7. Aida $Y$, Kudo 0 , Yamakawa $K$, et al. Papillary adenocarcinoma of the ureter producing carcinoembryonic antigen and carbohydrate antigen. J Urol 2002;168:2535-6. http://dx.doi.org/10.1016/S0022$5347(05) 64189-4$

8. Matsuoka $Y$, Ishimaru $H$, Arai $G$, et al. A case of transitional cell carcinoma of the renal pelvis with adenocarcinoma producing CEA and CA19-9. Acta Urol Jpn 2004:50:637-40.

9. Onishi T, Franco E0, Shibahara T, et al. Papillary adenocarcinoma of the renal pelvis and ureter producing carcinoembryonic antigen, carbohydrate antigen 19-9 and carbohydrate antigen 125. Int I Urol 2005;12:214-6. http://dx.doi.org/10.1111/i.1442-2042.2005.01009.x

10. Kobori Y, Shigehara K, Amano $T$, et al. Port site metastasis of primary adenocarcinoma of the renal pelvis after laparoscopic nephrectomy: a case report. Acta Urol Jpn 2005;51:105-8.

11. Azumi M, Hou K, Numata A, et al. Case report of renal pelvic adenocarcinoma associated with a renal stone that produced carbohydrate antigen 125 and carbohydrate antigen 19-9. Acta Urol Jpn 2007;53:631-4.

12. Shih $\mathrm{CM}$, Huang $\mathrm{CT}$, $\mathrm{Chi} \mathrm{CH}$, et al. CA125-producing clear cell adenocarcinoma arising from the upper ureter and renal pelvis. J Chin Med Assoc 2010;73:40-3. http://dx.doi.org/10.1016/S1726$4901(10) 70020-4$

13. Kato M, Onishi T, Hoshina A, et al. Successful treatment with paclitaxel/carboplatin chemotherapy in advanced adenocarcinoma of the urinary tract producing carcinoembryonic antigen, carbohydrate antigen 19-9 and carbohydrate antigen 125. Urol Int 2010;84:116-8. http://dx.doi.org/10.1159/000273479

14. Ye YL, Bian J, Huang YP, et al. Primary mucinous adenocarcinoma of the renal pelvis with elevated CEA and CA19-9. Urol Int 2011;87:484-8. http://dx.doi.org/10.1159/000329767

Correspondence: Dr. Hai Jiang, Department of Urology, the First Affiliated Hospital, School of Medicine, Zhejiang University, Hangzhou 310003, China; fax:+86-571-8707-2577; jianghaizju@aliyun.com 